\title{
Quiste de duplicación esofágica. Informe de un caso y revisión de la literatura
}

\author{
Dra. Rosangela Del Razo, ${ }^{*}$ Dra. Airé Gómez, ${ }^{*}$ Dra. Margarita Salcedo, ${ }^{* *}$ Dr. Roberto Velásquez ${ }^{* *}$
}

\section{RESUMEN}

Los quistes congénitos del mediastino no son frecuentes; su prevalencia en la población adulta es mayor que en los niños. El quiste de duplicación esofágica es la segunda duplicación más frecuente del tubo digestivo después del íleo. Los quistes del intestino anterior (en la división embriológica del intestino primitivo hay intestino anterior y posterior) son el resultado de una división anormal del intestino primitivo. Histológicamente son un saco muscular revestido de epitelio estratificado. La mayoría de estas anomalías son asintomáticas; en la mitad de los pacientes se descubren incidentalmente. En el abordaje diagnóstico del dolor torácico, los quistes del tubo digestivo son poco frecuentes; sin embargo, la fiebre y el dolor agudo pueden ser debidos a maceración o a su ruptura. Se ha descrito su asociación con anormalidades de la columna vertebral como espina bífida, hemivértebras o defectos de la fusión vertebral.

Palabras clave: Quiste de mediastino, íleo, saco muscular, epitelio estratificado, espina bífida.

\section{ABSTRACT}

Congenital cysts of the mediastinum are infrequent; their prevalence in the adult population is higher than in children. An esophageal duplication cyst is the second more frequent duplication of the gastrointestinal tract after ileal duplications. Cysts of the foregut result from an abnormal division of the primitive intestine. Histologycally, a cyst is a muscular sac lined with stratified epithelium. It is usually asymptomatic and in half of the patients it is an incidental finding. In the aetiology chest pain, a cyst of the digestive tract is a rare occurrence. On the other hand fever and acute pain can be caused by maceration or rupture of the cyst. Cysts have been associated with spinal abnormalities such as spina bifida, hemivertebrae and vertebral fusion defects.

Key words: Mediastinum cyst, ileum, muscular coat, stratified epithelium, bifid thorm.

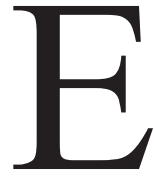

1 quiste de duplicación esofágica es un saco muscular revestido de epitelio estratificado que se recubre de epitelio respiratorio, gástrico o intestinal. Los quistes del intestino anterior son el resultado de una gemación o división anormal del intestino primitivo. Los quisten congénitos del mediastino, no son frecuentes. Los quistes del intestino anterior, incluyendo

* Residentes de segundo año de Neumología Pediátrica, Instituto Nacional de Enfermedades Respiratorias

** Médico Adscrito al Servicio de Neumología Pediátrica, Instituto Nacional de Enfermedades Respiratorias

Correspondencia: Dra. Rosangela del Razo. Instituto Nacional de Enfermedades Respiratorias. Calz. de Tlalpan No. 4502. Col. Toriello Guerra. Del. Tlalpan. Tel: 56653958. rosangelarr@yahoo.com

Recibido: agosto, 2009. Aceptado: abril, 2010.

Este artículo debe citarse como: Del Razo R, Gómez A, Salcedo M y col. Quiste de duplicación esofágica. Informe de un caso y revisión de la literatura. Acta Pediatr Mex 2010;31(3):108-114.

www.nietoeditores.com.mx los broncogénicos y los esofágicos, representan $48.6 \%$ de todos los quistes del mediastino. Los quistes broncogénicos son más comunes que los esofágicos. Estos quistes de duplicación ocurren en uno de cada 8,200 pacientes. La mayoría de estas lesiones son asintomáticas, pero pueden causar disfagia por compresión, dolor por perforación, sangrado y degeneración maligna. En la mitad de los pacientes se descubren incidentalmente. En un paciente con un quiste de duplicación esofágica que experimenta fiebre y dolor torácico hay que sospechar que ha ocurrido maceramiento o ruptura del quiste en el mediastino.

Se recomienda la excisión quirúrgica de las lesiones quísticas pulmonares. Se ha mencionado en la literatura $10 \%$ de mortalidad operatoria y postoperatoria.

Informamos el caso de una paciente de 14 años, con cuadro clínico de seis meses de evolución, con dolor torácico como síntoma fundamental. El estudio se hizo pensando en una masa mediastinal. El estudio histopatológico estableció que se trataba de un quiste por duplicación esofágica. 


\section{PRESENTACIÓN DEL CASO}

Niña de 14 años, previamente sana, con un padecimiento de seis meses de evolución. Inició su problema con disnea progresiva, hiporexia, dolor en región torácica baja derecha, progresivo de tipo pleurítico; pérdida de peso de $4.5 \mathrm{~kg}$ en cuatro meses ( $10 \%$ del peso corporal total), palpitaciones, astenia y adinamia.

Examen físico. Signos vitales: frecuencia cardiaca, frecuencia respiratoria, tensión arterial, temperatura en límites normales; talla menor a porcentila 3 para la edad y para el peso. Cuello corto; tórax asimétrico a expensas de hemitórax derecho; la amplexión y la amplexación revelaron movimientos del hemotórax derecho disminuidos: vibraciones vocales disminuidas. La transmisión de la voz y la auscultación de los ruidos respiratorios estaban disminuidos en este hemitórax y abolidos en la base; había matidez basal a la percusión. No existían datos de dificultad respiratoria. Se diagnosticó un síndrome de derrame pleural. Los estudios de laboratorio y gabinetes iniciales mostraron biometría hemática con leucocitos normales para la edad, con neutrofilia. La radiografía de tórax posteroanterior mostraba gran opacidad homogénea en el hemitórax derecho, con "signo de la silueta" positivo, que no permitía definir el hemidiafragma ipsilateral, ya que desplazaba las estructuras mediastinales hacia el lado opuesto (Figura 1). La radiografía lateral de tórax mostró una imagen que sugería su localización en el mediastino medio o posterior.

La tomografía de tórax contrastada, con ventana para mediastino, (que permite evaluar estructuras vasculares y ganglios) mostró una lesión en el mediastino medio; era redondeada, con bordes bien definidos; medía 12 x $17 \mathrm{~cm}$ de diámetro y se hallaba contigua a la arteria pulmonar. $\mathrm{Su}$ imagen era heterogénea: predominaba la densidad líquida y en su interior revelaba múltiples lesiones pequeñas, redondeadas, con la densidad de la grasa (Figura 2). En la ventana para parénquima se confirmó que la lesión era dependiente del mediastino. El parénquima pulmonar no mostró alteraciones. Para definir mejor el contenido líquido de la lesión se realizó ultrasonido de tórax y abdomen, donde se vio una masa de contornos bien definidos, de aspecto lobulado y contenido heterogéneo debida a múltiples imágenes redondeadas ecogénicas, bien definidas, sin vascularidad, similares a la grasa, la mayor de $30 \mathrm{~mm}$. El Doppler color, mostró una imagen anecóica con ecos finos en su interior. El estudio de marcadores tumorales

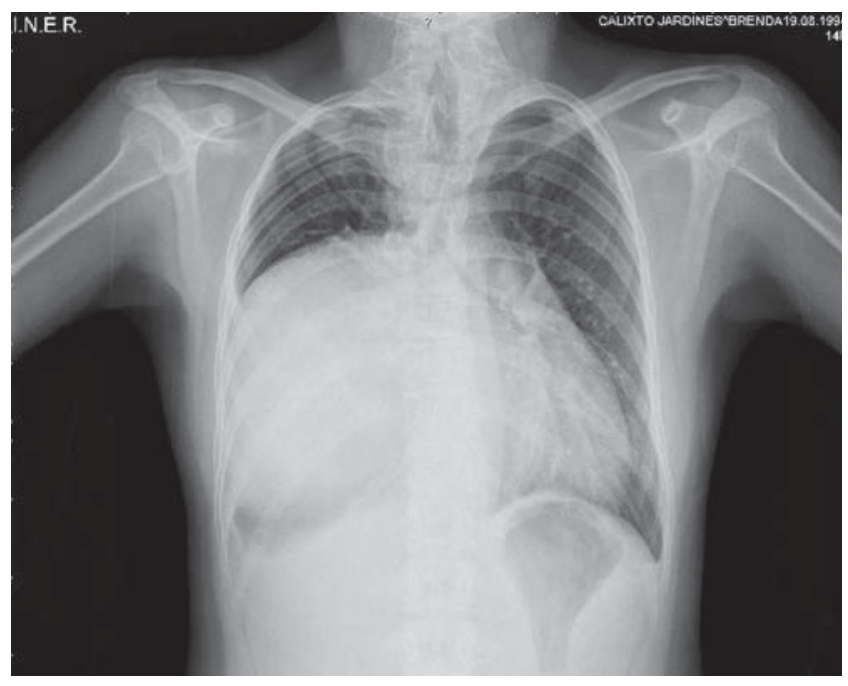

Figura 1. Radiografía PA de tórax de la paciente, que muestra radiopacidad homogénea en el hemitórax derecho proveniente de mediastino.

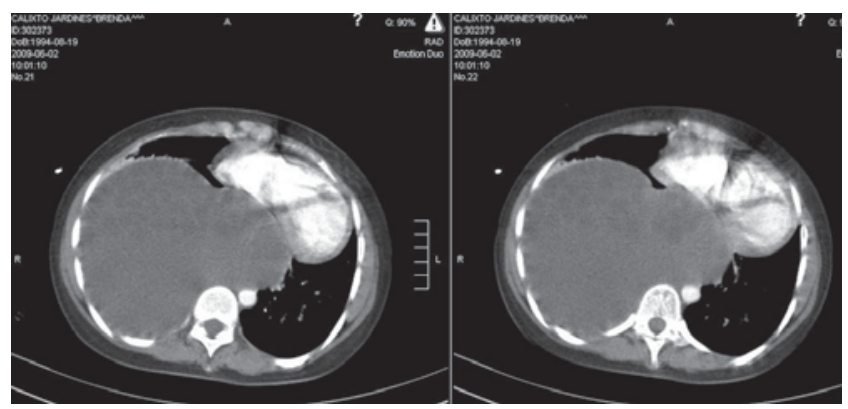

Figura 2. TAC de tórax simple y contrastada de la misma paciente que muestra una lesión dependiente del mediastino, redondeada, con aparente densidad líquida y lesiones quísticas en su interior.

con hormona gonadotropina coriónica, alfafetoproteína y antígeno carcinoembrionario dio resultados negativos.

Debido a la persistencia de la disnea y al contenido predominantemente líquido de la lesión se consultó al Servicio de Cirugía. Se decidió realizar una toracocentesis diagnóstica. Se obtuvieron $25 \mathrm{~mL}$ de líquido de aspecto lechoso con grumos blancos; el análisis citoquímico informó que era un líquido de aspecto purulento, con coágulos. No se realizó análisis químico, pues el líquido era demasiado espeso; sólo se refirió que tenía $100 \%$ de polimorfonucleares y había bacterias. Estudio anatomopatológico: frotis de líquido con material proteináceo, detritus celulares, abundantes polimorfonucleares, algunos macrófagos así como linfocitos; se aprecian escasas escamas y células pavimentosas. Diagnóstico: líquido compatible con empiema. 
La paciente fue sometida a una toracotomía posterolateral derecha, durante la cual se encontró líquido turbio en la cavidad torácica, no fétido, aproximadamente $300 \mathrm{cc}$; el lóbulo inferior se hallaba colapsado y tenía una lesión quística en la región basal; desplazaba el esófago hacia la región anterior; existían adherencias con el esófago. Había contenido sebáceo en el interior de la lesión.

Descripción macroscópica de la pieza quirúrgica quística: Masa de $660 \mathrm{~g}$; medía 13 × 8 × $6 \mathrm{~cm}$; la superficie externa es lisa, con trayectos vasculares congestivos en casi toda su extensión; opaca y cubierta por adherencias fibrinopurulentas y hemorrágicas; en uno de sus bordes tiene un segmento de contornos irregulares, grueso, laminado y vascularizado. Al corte, el contenido es pastoso y filante; el revestimiento es café blanquecino, granular y opaco. El fragmento relatado recuerda al esófago en su estructura mural con la adventicia hemorrágica, congestión transmural y erosión focal de la mucosa; la descripción microscópica se omitió por el Servicio de Patología. Diagnóstico: quiste mediastinal: quiste del intestino anterior de tipo quiste esofágico (duplicación), ulcerado, con inflamación xantogranulomatosa (queratina). Mediastinitis aguda inespecífica y crónica con tejido de granulación, reacción xantogranulomatosa y hemorrágica reciente y antigua (hemosiderosis).

Una broncoscopia mostró anatomía normal, escasas secreciones mucohialinas. La SEGD realizada para descartar alteraciones a nivel digestivo reveló dilatación esofágica a nivel de su tercio medio.

Las radiografías AP y lateral de columna cervical muestran diástasis total de cuerpo vertebral C5 (Figura 3).

En la radiografía de tórax postoperatoria se observa adecuada reexpansión pulmonar y dilatación del esófago, que pudo observarse con mayor claridad en fluoroscopia.

\section{DISCUSIÓN}

Embriología. El sistema respiratorio se desarrolla de la pared ventral del intestino anterior entre la tercera y cuarta semanas (periodo embriológico) de la gestación, y continúa su desarrollo durante los dos primeros años de vida. Durante dicho periodo, el primordio del pulmón es un divertículo del intestino anterior cerca de las siete semanas. El epitelio de la tráquea, bronquios y alvéolos

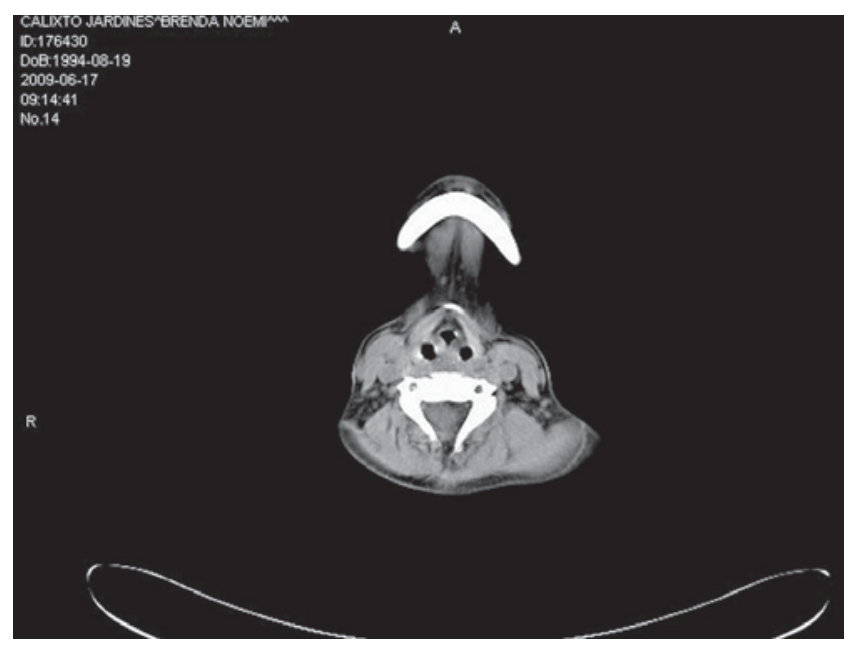

Figura 3. TAC de cuello de la paciente que muestra diástasis total de cuerpo vertebral C5.

se origina del endodermo ${ }^{1,2}$; los músculos, cartílagos y tejido conectivo, del mesodermo.

El árbol traqueobronquial se desarrolla entre los días 24 a 36 de gestación; se inicia a los 24 a 26 días con una protuberancia medial de la parte ventral de la faringe que se desarrolla al final de la ranura laringotraqueal. Entre los días 26 y 28 , la protuberancia dará origen a las gemas pulmonares derecha e izquierda ${ }^{3}$. Posteriormente, la invaginación del mesodermo constituye el septum traqueoesofágico que separa el esófago de la tráquea ${ }^{3,4}$.

Epidemiología. Las anormalidades esofágicas congénitas son relativamente comunes: 1:3000 a 1:4500 recién nacidos vivos ${ }^{5}$.

E1 30 a $40 \%$ de las masas mediastinales pediátricas, se desarrolla en el mediastino posterior ${ }^{6}$. Los quistes congénitos del mediastino no son frecuentes; representan 12 al $18 \%$ de las masas mediastinales ${ }^{7,8}$.

Nakahara et al, estudiaron 580 tumores mediastinales; sólo dos fueron duplicaciones esofágicas ${ }^{10}$.

Takeda $\mathrm{S}$ et al ${ }^{9}$ revisaron los casos de un hospital universitario que hubo en 50 años. Encontraron que la prevalencia de quistes en la población adulta es mayor que en niños ( $14.1 \%$ vs $7.7 \%$ con p significativa). Los quistes del intestino anterior, incluyendo los broncogénicos y esofágicos, representan $48.6 \%$ de todos los quistes mediastinales. Los quistes broncogénicos son más comunes que los esofágicos. En la población estudiada, hubo tres quistes esofágicos y sólo uno fue en un niño.

Se ha descrito que estos quistes de duplicación ocurren entre 8,200 pacientes ${ }^{11}$, aunque es la segunda duplicación 
más frecuente del tubo digestivo después de la del íleo; comprende del 15 al $20 \%{ }^{12-15}$.

Ildstad ST ${ }^{16}$, halló 20 duplicaciones entéricas en un hospital pediátrico en 30 años; fueron más frecuentes en menores de dos años (límites de un día a 11 años); seis fueron esofágicas y predominaron en niñas; tres de ellas tenían otras malformaciones congénitas y otras tres tenían una segunda duplicación del tubo digestivo.

Cioffi $\mathrm{U}^{17}$, describió 27 casos de quistes mediastinales, 11 de duplicación esofágica (dos en menores de 30 años) y 16 broncogénicos.

\section{Clasificación}

La clasificación de los quistes mediastinales se basa en su etiología; se incluyen quistes broncogénicos, duplicación esofágica originada en el intestino anterior, quistes mesodérmicos que originan quistes pericárdicos o pleurales, quistes tímicos y quistes misceláneos. Estas entidades son malformaciones broncopulmonares del intestino anterior; pueden ser secuestro pulmonar, malformación adenomatoidea quística, enfisema lobar congénito y quiste broncogénico. Los quistes neuroentéricos pueden desarrollarse por migración anormal de las células germinales broncogénicas, las cuales se mezclan con la columna vertebral, en la formación del canal neuroentérico primitivo de la notocorda.

Los quistes mesoteliales incluyendo los quístes pericárdicos, los pleurales, los tímicos y otros más raros pueden ocurrir en el mediastino ${ }^{10}$.

Embriológicamente son de tres clases ${ }^{18}$ :

Broncogénicos, esofágicos intramurales (duplicación verdadera), entéricos.

\section{Fisiopatogenia}

Se han propuesto varias teorías para explicar la base embriológica de las duplicaciones intestinales. Se cree que los quistes del intestino anterior son el resultado de una gemación o división anormal del intestino primitivo ${ }^{10}$.

La teoría de la recanalización aberrante del lumen propuesta por Bremer ${ }^{19}$ es adecuada para explicar las duplicaciones en las partes del tubo digestivo que pasan por la "fase sólida", como son el esófago y el colon. En la 5ta y 6ta semanas de vida intrauterina, el intestino anterior es cubierto por células similares a las del aparato respiratorio. Este epitelio crece y oblitera el lumen; posteriormente produce secreciones que forman vacuolas en el espacio intracelular. Las vacuolas se alínean longitudinalmente y eventualmente se unen para formar un nuevo lumen. $\mathrm{Si}$ por alguna razón las vacuolas fallan en la coalición en el eje longitudinal, se formará un quiste que puede migrar lateralmente en la pared del esófago y se rodea de fibras musculares.

Debido a la elongación de las vísceras y la dextrorotación intratorácica del estómago, estos quistes frecuentemente aparecen en la porción inferior derecha del esófago.

El segmento duplicado tiene una pared delgada de músculo y se alínea con la mucosa de tubo digestivo. La mayoría de los quistes esféricos (redondos) deriva de un divertículo verdadero del que son proyectados desde la superficie ventral antimesentérica ${ }^{20} ; 80 \%$ de estas duplicaciones forma quistes que usualmente son estructuras únicas llenas de líquido ${ }^{21}$.

\section{Histología}

Para que un quiste se considere como esofágico, debe estar parcial o totalmente cubierto por epitelio escamoso estratificado, con pared muscular y fibrosa ${ }^{7}$. La diferencia entre quiste broncogénico y quiste de duplicación esofágica es la presencia de cartílago lo que sugiere un origen broncogénico ${ }^{10}$.

La duplicación completa es una malformación rara, frecuentemente asociada con duplicación gástrica. La visualización del segmento duplicado en su forma tubular completa, depende de su comunicación con el esófago o el estómago normales y puede ser reconocido hasta después de la cirugía ${ }^{23}$.

\section{Cuadro clínico}

Hasta $75 \%$ de estas lesiones son asintomáticas ${ }^{10}$. Las manifestaciones clínicas pueden deberse a la compresión del árbol traqueoesofágico ${ }^{7}$ : disfagia, dolor por perforación, sangrado y degeneración maligna ${ }^{10}$. En la mitad de los pacientes se descubren incidentalmente ${ }^{9}$. En las causas de dolor torácico, el origen digestivo es poco frecuente en niños. Las manifestaciones clínicas dependen de la situación anatómica, la relación con las estructuras vecinas y el tamaño; pueden infectarse, supurar y formar una fístula ${ }^{22}$. Ocasionalmente puede haber comunicación entre el quiste y el bronquio, y en varios casos el quiste esofágico puede estar en la pared muscular del esófago ${ }^{7}$. 
Takeda S et al ${ }^{9}$ estudiaron 105 pacientes; diez eran niños. El 36.2\% fue sintomático; 39.2\% de los quistes era de intestino anterior brocogénicos o esofágicos.

Los síntomas más frecuentes fueron dolor retroesternal (14.3\%), disnea (7.6\%), tos (6.7\%) y fiebre (5.7\%).

Dakshersh ${ }^{24}$ estudió 22 niños de $7.7 \pm 2.2$ años de edad con quistes torácicos. Los principales síntomas fueron tos y disnea (100\%), episodios de cianosis (58\%), disfagia (55\%), dolor torácico (46\%), hemoptisis (18\%) detención del crecimiento (55\%) y dolor pleurítico (18\%). Tres niños tuvieron quiste de duplicación esofágica que involucraba del lóbulo superior derecho.

Existen pocos informes sobre el tamaño de las lesiones: Bremer $\mathrm{J}{ }^{20}$ menciona que pueden ser pequeñas, como bandas verticales que cruzan el lumen de una pared a otra. Nakahara $\mathrm{K}$ et al describieron el caso de un hombre de 36 años, cuya radiografía mostraba una masa mediastinal de 4 x $4 \mathrm{~cm}$, sin desviación de la carina ni del esófago; sin embargo, con una esofagofibroscopia se hallaron dos fístulas de $7 \mathrm{~mm}$ de diámetro. Durante una operación se vio que el quiste se extendía desde la carina hasta $7 \mathrm{~cm}$ hacia abajo, y estaba adherido al esófago con $4 \mathrm{~cm}$ de base ${ }^{9}$. Arbona et al informaron el caso de una mujer de 24 años, en quien una serie gastrointestinal alta reveló una masa de $3.5 \mathrm{~cm}$; al ser resecado se vio que medía 2 $\mathrm{x} 2 \times 1.3 \mathrm{~cm}^{25}$.

Complicaciones y asociaciones. La mayoría de estas lesiones es asintomática, pero pueden causar complicaciones: perforación, sangrado, compresión ${ }^{11}$. En un paciente que tenga quiste de duplicación esofágica y curse con fiebre y dolor torácico agudo puede pensarse que se produjo maceramiento o ruptura del quiste en el mediastino ${ }^{9}$.

Las lesiones quísticas pueden infectarse, secretar moco, mostrar incremento de la proliferación celular y disminución de la apoptosis ${ }^{24}$.

El quiste de duplicación predispone a reflujo gastroesofágico y a la impactación de cuerpos extraños.

Las anomalías asociadas con quiste de duplicación esofágica halladas por Kitano $\mathrm{Y}^{26}$ fueron quiste broncogénico intrapulmonar, secuestro intrapulmonar, bronquiectasias y hubo comunicación del quiste de duplicación esofágica con lesiones quísticas pulmonares coexistentes en la mayoría de los pacientes.

Los bronquios aberrantes pueden coexistir con malformaciones quísticas pulmonares ${ }^{3}$.
La asociación entre duplicaciones esofágicas y atresia esofágica o fístula traqueoesofágica es rara pero tiene un potencial de complicaciones elevado ${ }^{11}$.

\section{Diagnóstico}

El diagnóstico del quiste de duplicación esofágica en la mayoría de los casos es incidental en el examen de una radiografía de tórax, en $37 \%$ de los casos ${ }^{27}$.

Los quistes mediastinales benignos frecuentemente se sospechan por sus características en una tomografía computada; su diagnóstico y tratamiento pueden realizarse con técnicas quirúrgicas mínimamente invasivas ${ }^{8}$.

La mayoría de los quistes son esféricos; rara vez hacen una impresión sobre el esófago y usualmente se localizan en el hemitórax derecho; ocurren en la porción media y baja del esófago ${ }^{11,21}$. Radiológicamente se ven como masas mediastinales posteriores ${ }^{13}$, aunque los quistes benignos suelen estar en el mediastino medio ${ }^{8}$.

La esofagoscopia con bario se ha realizado sobre todo en pacientes con sospecha de quistes esofágicos o broncogénicos ${ }^{10}$. En los quistes de duplicación intestinal, el esofagograma muestra el esófago desplazado hacia el sitio opuesto al quiste o una masa intramural extramucosa ${ }^{25}$. En la TAC, la duplicación es una masa de densidad homogénea parecida a la del agua y no es mayor después de la inyección de material de contraste intravenoso ${ }^{15}$. Con la RMN, la mayoría de las duplicaciones tiene una baja señal de intensidad en T1 y alta intensidad en T2 ${ }^{28}$. El ultrasonido endoscópico puede mostrar contigüidad de la muscularis propia del esófago que continúa con el músculo en la pared del quiste ${ }^{25}$.

\section{Diagnóstico diferencial}

El diagnóstico diferencial debe hacerse con cualquier masa en el mediastino posterior o medio, como las neoplasias de la cadena simpática, los quistes broncogénicos y neuroentéricos, el secuestro pulmonar, el meningocele anterior o el hemangioma; tumores extramucosos intramurales del esófago como leiomioma, lipoma, fibrolipoma, neurofibroma ${ }^{14,25}$.

El quiste de duplicación intestinal tiene una gran porción de su pared con todas las capas intestinales pues es una parte del tubo original ${ }^{20}$, lo que no ocurre en un divertículo.

\section{Tratamiento}

Las lesiones quísticas pulmonares que no responden al tratamiento médico deben operarse ${ }^{24}$. 
La resección de las lesiones quísticas crónicamente inflamadas puede causar sangrado perioperatorio, sepsis, laceración esofágica, úlcera esofágica, hemorragia, absceso intraparenquimatoso y falla respiratoria. Se ha señalado $10 \%$ de mortalidad por estas complicaciones ${ }^{29}$. Cuando es necesario hacer una resección muy extensa, puede requerirse una reconstrucción esofágica ${ }^{16}$.

Las lesiones quísticas del pulmón pueden remitir gracias a que su crecimiento es diferente al del parénquima circundante, dando la falsa apariencia de que la lesión disminuye de tamaño ${ }^{30}$.

\section{CONCLUSIÓN}

La mayoría de los quistes de duplicación esofágica son lesiones asintomáticas. Nuestra paciente tuvo síntomas a los 14 años de edad; aparecieron seis meses antes de su ingreso. El principal fue el dolor torácico. Hay que señalar que el dolor de etiología digestiva es poco frecuente en niños. Luego apareció disnea, que al igual que en la revisión de Takeda $\mathrm{S}$ et al es el segundo síntoma más frecuente.

Los quistes congénitos son infrecuentes y más aún los esofágicos, cuya clasificación se basa en la embriología; su diagnóstico es histológico. El abordaje de masas medistinales es muy extenso; se debe realizar una serie esofagogastroduodenal para descartar causas digestivas.

En este caso llamaron la atención las características por estudio de imagen, debido a que se trataba de una gran masa heterogénea con múltiples imágenes redondeadas en su interior. Con la radiografía de tórax se encontró una masa mediastinal, entre las posibilidades diagnósticas era un quiste broncogénico, un secuestro pulmonar (ambos con proceso infeccioso activo) o un divertículo; fueron descartadas cuando se sometió a un procedimiento quirúrgico y se realizó el estudio histopatológico.

El tamaño de la lesión de nuestro caso fue mucho mayor a lo que menciona la literatura $(17 \mathrm{~cm}$ vs $4 \times 4 \mathrm{~cm}$ vs $2 \times 2 \times 1.3 \mathrm{~cm}$ vs menores de $5 \mathrm{~cm}$ ) en pacientes de edad mayor. La mediastinitis y la infección del contenido del quiste fueron propiciadas por la comunicación que tenía con el esófago. La resección quirúrgica se llevó a cabo sin contratiempos, a pesar de que es una cirugía con riesgo de sangrado profuso, sepsis, laceración esofágica, abscesos, etc.

\section{Agradecimientos}

Al Doctor Alejandro Alejandre, Jefe del Servicio de Neumología Pediátrica y a los Servicios de Radiología, Cirugía de Tórax y Patología del Instituto Nacional de Enfermedades Respiratorias por su apoyo en la elaboración del presente trabajo y en el diagnóstico del caso.

\section{REFERENCIAS}

1. Keslar P, Newman B, Oh KS. Radiographic manifestation of anomalies of the lung. Radiol Clin North Am. 1991;29:255-70. citado en Berrocal T. Congenital Anomalies of the Tracheobronchial Tree, Lung, and Mediastinum: Embryology, Radiology, and Pathology. Radiographics. 2003;24:17.

2. Skandalakis JE, Gray SW, Symbas P. The trachea and the lung. En: Skandalakis JE, Gray SW, eds. Embryology for surgeons. 3rd ed. Baltimore, Md: Williams \& Wilkins; 1994. p. 414-50 citado en Berrocal T. Congenital Anomalies of the Tracheobronchial Tree, Lung, and Mediastinum: Embryology, Radiology, and Pathology. Radiographics. 2003;24:17.

3. Ghaye B, Szapiro D, Fanchmps JM, Dondelinger RF. Congenital bronchial abnormalities revisited. Radiographics. 2001;21:105-19.

4. Burri PH. Fetal and postnatal development of the lung. Annu Rev Physiol. 1984;46:617-28. citado en Berrocal T. Congenital Anomalies of the Tracheobronchial Tree, Lung, and Mediastinum: Embryology, Radiology, and Pathology. Radiographics. 2003;24:17.

5. Yazbeck S. Gastrointestinal emergencies of the neonate. En: Roy CC, Silverman A Alagille D, ed. Pediatric Clinical Gastroenterology. $4^{\text {th }}$ ed. St Louis Mosby-Year Book; 1995 .p.53.

6. Franco A. Diagnóstico por imagen de las masas mediastínicas en pediatría. Radiol Clin North Am. 2005;43:325-53.

7. Wychulis AR, Payne WS, Clagett OT, et al. Surgical treatment of mediastinal tumors: a 40 year experience. J Thorac Cardiovasc Surg. 1971;62:379-92.

8. Whooley BP, Urschel JD, Antkowiak JG, et al. Primary tumors of the mediastinum. J Surg Oncol. 1999;70:95-9.

9. Takeda S, Miyoshi S, Minami M, Ohta M, Masaoka A, Matsuda $\mathrm{H}$. Clinical spectrum of mediastinal cysts. Chest. 2003;124:125-32.

10. Nakahara K, Fujii Y, Miyoshi S, et al. Acute symptoms due to a huge duplication cyst ruptured into the esophagus. Ann Thorac Surg. 1990;50:309-11.

11. Snyder C. Esophageal duplication cyst UIT esophageal web and tracheoesophageal fistula. J Pediatr Surg. 1996;31:968-9.

12. Benson JE, Olsen MM, Fletcher BD. A spectrum of bronchopulmonary anomalies associated with tracheoesophageal malformations. Pediatr Radiol. 1985;15:377-80.

13. Berrocal T, Torres I, Gutiérrez J, Prieto C, del Hoyo ML, Lamas $\mathrm{M}$. Congenital anomalies of the upper gastrointestinal tract. Radiographics. 1999;19:855-72.

14. Kirkpatrick JA, Wagner ML, Pilling GPA. A complex of anomalies associated with tracheoesophageal fistula and esophageal atresia. Am J Roentgenol. 1965; 95:208-12.

15. Macpherson RI. Gastrointestinal tract duplications: clinical, pathologic, etiologic, and radiologic considerations. Radiographics. 1993;13:1063-80. 
16. Ildstad ST, Tollerud DJ, Weiss RG, Ryan DP, McGowan MA, Martin LW. Duplications of the alimentary tract: clinical characteristics, preferred treatment and associated malformations. Ann Surg. 1988;208:184-9.

17. Cioffi U. Presentation and surgical management of broncogenic and esophageal duplication cyst in adults. Chest. 1996;113:1492-6.

18. Kirman WO. Cystic intrathoracic derivatives of the foregut and their complications. Thorax. 1973;29:424-8 citado en Nakahara $\mathrm{K}$, Fujii Y, Miyoshi S, et al. Acute symptoms due to a huge duplication cyst ruptured into the esophagus. Ann Thorac Surg. 1990;50:309-11.

19. Plavsic BM, Robinson AE, Jeffrey RB, Jr. Gastrointestinal Radiology. New York, NY: McGraw-Hill; 1992.p.612-28. citado en Berrocal T. Congenital Anomalies of the Tracheobronchial Tree, Lung, and Mediastinum: Embryology, Radiology, and Pathology. Radiographics. 2003;24:17.

20. Bremer JL. Diverticula and duplications of the intestinal tract. Arch Pathol. 1944; 38:132-40.

21. Gonzalez Z. Malformaciones gastrointestinales: causa de dolor torácico. An Pediatr (Barc). 2007;66(3):309-12.

22. Fenoglio-Preiser $\mathrm{CM}$. The normal anatomy of the esophagus. En: Gastrointestinal Pathology: An atlas and text. $2^{\text {nd }}$ ed. Philadelphia: Lippincott-Raven Publishers; 1999. p. 15

23. Hertzberg BS, Bowie JD. Fetal gastrointestinal abnormalities.
Radiol Clin North Am. 1990;28:101-14, citado en Berrocal T. Congenital Anomalies of the Tracheobronchial Tree, Lung, and Mediastinum: Embryology, Radiology, and Pathology. Radiographics. 2003;24:17.

24. Dakshersh P. Congenital cystic lung lesions: is surgical resection essential?. Pediatric Pulmonol. 2005;40:533-7.

25. Arbona JL, Fazzi JGF, Mayoral J. Congenital esophageal cysts: case report and review of the literature. Am J Gastroenterol. 1984;79:177-82.

26. Kitano Y. Esophageal duplication cyst associated with pulmonary cystic malformations. J Pediatr Surg. 1995;30:1724-7.

27. Bower RJ, Sieber WK, Kiesewetter WB. Alimentary tract duplications in children. Ann Surg. 1978;188:689-94 citado en Berrocal T. Congenital Anomalies of the Tracheobronchial Tree, Lung, and Mediastinum: Embryology, Radiology, and Pathology. Radiographics. 2003;24:17.

28. Ribet ME, Copin MC. Bronchogenic cysts of the lung. Ann Thorac Surg. 1996;61:1636-40.

29. Nobuhara KK. Bronchogenic cysts and oesophageal duplications: common origins and treatment. J Pediatr Surg. 1997;32:1408-13.

30. Roggin KK. The unpredictable character of congenital cystic lung lesions. J Pediatr Surg. 2000;35:23-33 citado en Dakshersh P. Congenital cystic lung lesions: is surgical resection essential?. Pediatric Pulmonol. 2005;40:533-7. 